\title{
Effect of Vagus Nerve Integrity on Short and Long-Term Efficacy of Antireflux Surgery
}

S. van Rijn, MD¹,2, N.F. Rinsma, MD¹, M.Y.A. van Herwaarden-Lindeboom, MD, PhD ${ }^{3}$, J. Ringers, MD , H.G. Gooszen, MD, PhD ${ }^{5}$, P.J.J. van Rijn, $\mathrm{MD}^{6}$, R.A. Veenendaal, MD, PhD , J.M. Conchillo, MD, PhD¹, N.D. Bouvy, MD, PhD² and Adrian A.M. Masclee, MD, PhD ${ }^{1}$

OBJECTIVES: Vagus nerve injury is a feared complication of antireflux surgery (ARS) that may negatively affect reflux control. The aim of the present prospective study was to evaluate short-term and long-term impact of vagus nerve injury, evaluated by pancreatic polypeptide response to insulin-induced hypoglycemia (PP-IH), on the outcome of ARS.

METHODS: In the period from 1990 until 2000, 125 patients with gastroesophageal reflux disease (GERD) underwent ARS at a single center. Before and 6 months after surgery, vagus nerve integrity testing (PP-IH), 24-h pH-monitoring, gastric emptying, and reflux-associated symptoms were evaluated. In 2014, 14-25 years after surgery, 110 patients were contacted again for evaluation of long-term symptomatic outcome using two validated questionnaires (Gastrointestinal Symptom Rating Scale (GSRS) and GERD-Health Related Quality of Life (HRQL)).

RESULTS: $\quad$ Short-term follow-up: vagus nerve injury (PP peak $\leq 47 \mathrm{pmol} / \mathrm{l})$ was observed in 23 patients (18\%) 6 months after fundoplication. In both groups, a comparable decrease in reflux parameters and symptoms was observed at 6-month follow-up. Postoperative gastric emptying was significantly delayed in the vagus nerve injury group compared with the vagus nerve intact group. Long-term follow-up: patients with vagus nerve injury showed significantly less effective reflux control and a higher re-operation rate.

CONCLUSIONS: Vagus nerve injury occurs in up to $20 \%$ of patients after ARS. Reflux control 6 months after surgery was not affected by vagus nerve injury. However, long-term follow-up showed a negative effect on reflux symptom control and re-operation rate in patients with vagus nerve injury.

Am J Gastroenterol 2016; 111:508-515; doi:10.1038/ajg.2016.42; published online 15 March 2016

\section{INTRODUCTION}

Gastroesophageal reflux disease (GERD) is a common health problem that affects $10-20 \%$ of individuals in Western society (1). Antireflux surgery (ARS) is indicated for patients with documented GERD and chronic symptoms refractory to proton-pump inhibitors (PPIs) or not willing to take lifelong PPIs. The aim of ARS is an optimal long-term control of reflux symptoms and reflux signs, without or with minimal side effects.

Despite improvements in surgical techniques such as laparoscopic antireflux surgery $(2,3)$, postoperative complications still occur (4-9). Vagus nerve injury is a well-known complication of ARS. Abdominal vagus function cannot be measured or evaluated directly. Data on the prevalence of vagus nerve dysfunction after antireflux surgery are therefore based on the presence of symptoms such as diarrhea, nausea, and vomiting $(6,10,11)$. In the past, an indirect method has been developed to measure vagus (dys)function by measuring the response of plasma pancreatic polypeptide (PP) secretion to insulin-induced hypoglycemia, the insulin hypoglycemia (IH)-PP test $(12,13)$.

In a previous study by our group, vagus nerve injury measured by the IH-PP test was observed in $10 \%$ of GERD patients after laparoscopic hemi-fundoplication (14). In that study, no significant differences were observed in the short-term postoperative outcome-that is, symptom control, reflux recurrence, and delayed

${ }^{1}$ Division of Gastroenterology-Hepatology, NUTRIM Research Institute, Maastricht University Medical Center, Maastricht, The Netherlands; ${ }^{2}$ Department of General Surgery, Maastricht University Medical Center, Maastricht, The Netherlands; 'Department of Paediatric Surgery, Wilhelmina Children's Hospital,

University Medical Center Utrecht, Utrecht, The Netherlands; ${ }^{4}$ Department of General Surgery, Leiden University Medical Center, Leiden, The Netherlands;

${ }^{5}$ Department of OR/Evidence Based Surgery, Radboud University Medical Center Nijmegen, Nijmegen, The Netherlands; ${ }^{6}$ Department of General Surgery, Lange

Land Hospital Zoetermeer, Zoetermeer, The Netherlands; ' 7 Department of Gastroenterology and Hepatology, Leiden University Medical Center, Leiden,

The Netherlands. Correspondence: Adrian A.M. Masclee, MD, PhD, Division of Gastroenterology-Hepatology, NUTRIM Research Institute, Maastricht University

Medical Center, P. Debyelaan 25, 6229 HX Maastricht, The Netherlands. E-mail: a.masclee@mumc.nl

Received 5 August 2015; accepted 24 January 2016 
gastric emptying between patients with or without vagus nerve injury. It should, however, be noted that the number of patients in that study (14) as in other studies assessing vagus nerve injury after antireflux surgery was small, and follow-up of patients was only short (14-16).

Data on the impact of vagotomy on outcome of antireflux surgery have been obtained by Oelschlager et al. These investigators reported on the effect of intended vagus nerve injury on the outcome of antireflux surgery. During a mean follow-up period of 18 months, vagus nerve damage did not negatively influence the outcome of antireflux surgery, nor that of gastric emptying (17).

It should be beyond any doubt that vagus nerve dysfunction measured by PP response to IH (IH-PP) truly results from injury during ARS and not from other causes. Therefore, the test should be performed both before and after surgery. Up to now, vagus nerve function in ARS has not been systematically and prospectively evaluated in a large cohort.

Aim of our study was to evaluate both short- and long-term impact of vagus nerve injury as evaluated by the IH-PP test on reflux symptoms, reflux control, and quality of life after ARS. We hypothesize that ARS-related vagus nerve injury will have a negative outcome on symptoms, reflux control, gastric emptying, and quality of life both after short- and long-term follow-up. In the nineties of the past century, as part of clinical work-up, we prospectively evaluated vagus nerve function by PP response to IH in patients undergoing ARS at the Leiden University Medical Center. Only data from patients with vagus nerve testing both before and after ARS have been evaluated. At 6 months after surgery, objective and subjective reflux parameters were measured. In 2014, a follow-up of this prospective cohort for long-term efficacy has been performed with questionnaires on reflux symptoms and quality of life.

\section{METHODS}

In the period from 1990 until 2000, out of a larger group, 125 patients with GERD underwent ARS at the Leiden University Medical Centre with detailed evaluation on the effect of ARS on several parameters. Patients underwent esophageal manometry, 24-h pH-monitoring, gastric emptying, and vagus nerve integrity tests both prior and 6 months after surgery as clinical evaluation. Symptoms were evaluated using questionnaires. In 2014, up to 25 years after surgery patients were contacted again and two validated questionnaires evaluating typical and atypical GERD symptoms were sent. Of the initial 125 patients 110 were eligible for follow-up (Figure 1).

\section{Operation}

Several surgical procedures were performed as procedure of choice from 1990 until 2000. The selection of the procedure was based on time-dependent surgeon's preference, not on patient characteristics. Initially, in the early nineties, the open Belsey Mark IV procedure was standard (18). After laparoscopic surgery became available at the Leiden University Medical Centre in the year 1994, the laparoscopic Nissen (19), and later on the Toupet

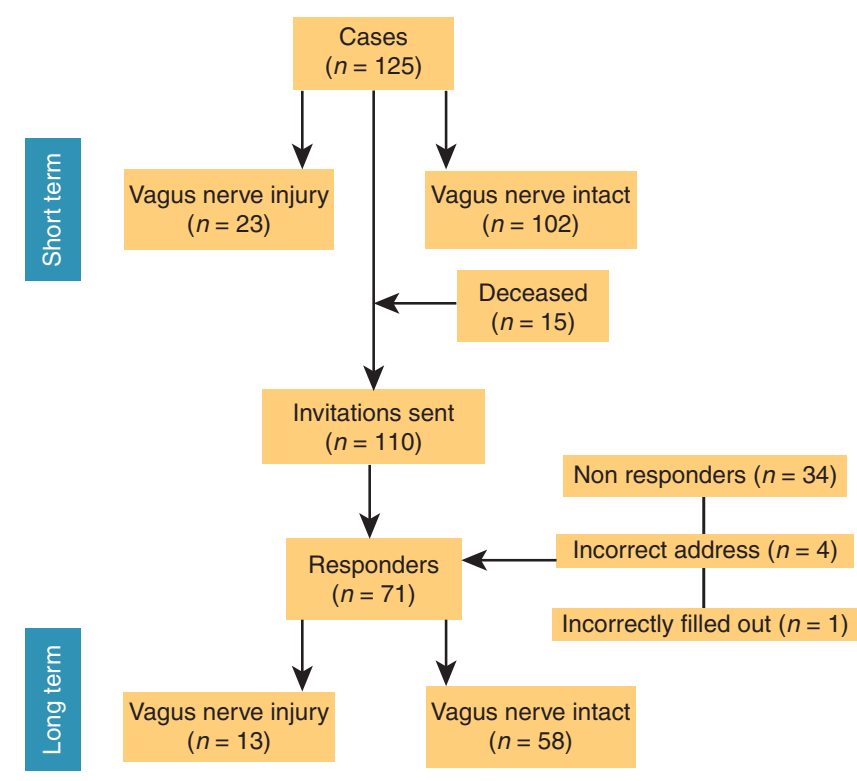

Figure 1. Cohort flow diagram.

fundoplication (20), became the procedure of choice based on literature data. In all patients, surgery was indicated based on documented GERD and symptoms refractory to medical therapy including PPIs.

\section{Short-term follow-up}

24-h $\boldsymbol{p H}$-monitoring. We performed 24-h ambulatory intraesophageal $\mathrm{pH}$-monitoring as previously described (4). Reflux parameters used in this study were the percentage of time with a $\mathrm{pH}$ level $<4$ for the total recording time, for time in the upright, and the supine position. Abnormal acid exposure time was defined as the percentage of time with $\mathrm{pH}<4$ for total time $\geq 4.0 \%$, upright time $\geq 4.4 \%$, and supine time $\geq 1.2 \%$ (21).

Esophageal manometry. Esophageal body and lower esophageal sphincter (LES) pressure recordings were obtained by a polyvinyl assembly with a 5-mm outer diameter incorporating a 6-cm-long sleeve sensor and seven side holes as described previously (9). The outcome variable used in this study was the LES mean pressure measured in $\mathrm{mm} \mathrm{Hg}$.

Gastric emptying. Gastric emptying was measured using a radionuclide scintigraphy with a dual-isotope method as previously described (14). Patients underwent gastric emptying testing pre- and postoperatively. Data of gastric emptying for solids were analyzed by determining lag phase, gastric emptying, and gastric half emptying time. The retention for liquids was calculated at 15 , 30,60 , and $90 \mathrm{~min}$. Gastric emptying for solids was considered normal if values for lag phase ranged from 13 to $31 \mathrm{~min}$ and for emptying rate from 36 to $79 \%$ per hour. Gastric emptying for solids was considered delayed if the emptying rate was $<35 \%$ per hour.

Vagus nerve integrity. As previously described, vagus nerve integrity was measured indirectly by the response of PP to insulin- 


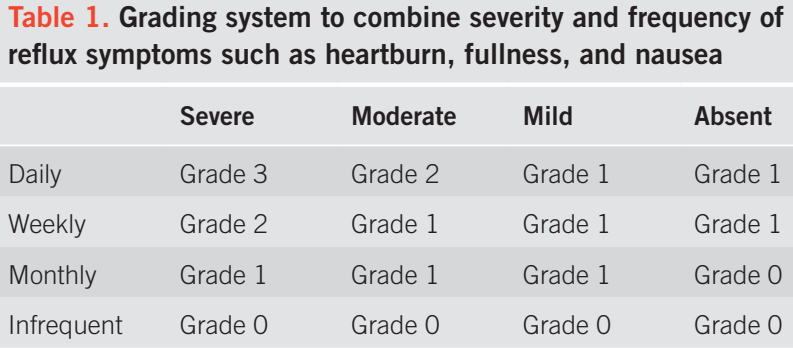

induced hypoglycemia (14). After an overnight fast, patients were administered a bolus of $0.1 \mathrm{U} / \mathrm{kg}$ of insulin (Actrapid, Novo Nordisk Farma BV, Bagsvaerd, Denmark) intravenously. After administration of insulin, blood samples were drawn at regular intervals during $150 \mathrm{~min}$ to measure PP and glucose levels. During the first $90 \mathrm{~min}$, blood was drawn with $10 \mathrm{~min}$ time intervals. At $90 \mathrm{~min}$, a standard test meal was given to the patients, and blood was withdrawn with 15 -min intervals up to $150 \mathrm{~min}$. PP levels were measured by a specific radioimmunoassay, as described previously (22). Nadir blood glucose levels in response to administration of insulin were $<2.5 \mathrm{mmol} / \mathrm{l}$ in all patients. A peak increment in plasma $\mathrm{PP} \leq 47 \mathrm{pmol} / \mathrm{l}$ was considered to be compatible with truncal vagotomy. Cutoff values calculated for this IH-PP test were based on data obtained from patients who underwent intentional vagotomy in comparison to a healthy control group (14). All participants in this study underwent vagus nerve integrity testing before and 6 months after surgery.

Symptoms. GERD symptoms were scored by a standardized symptom questionnaire before and 6 months after operation. Symptoms included heartburn, dysphagia, diarrhea, sensation of fullness, abdominal distention, vomiting, nausea, and belching. Severity and frequency of symptoms were combined according to a scoring system as shown in Table 1.

\section{Long-term follow-up}

In May 2014, two validated GERD-symptom questionnaires, the GERD-Health Related Quality of Life (HRQL) and Gastrointestinal Symptom Rating Scale (GSRS) symptom questionnaire, were sent out to the initial cohort.

We used these quantitative methods to evaluate symptom severity in GERD. Subjects were asked for severity and frequency of gastrointestinal symptoms (burning, bloating, belching, sensation of abdominal fullness, nausea, and pain) 14-25 years after surgery. The questionnaires assess typical and atypical GERD symptoms and patients' quality of life. In the GERD-HRQL questionnaire, patients were asked to indicate on a scale from 0 to 5 which number reflects best the severity of the symptom asked for in the question. The questionnaire has a minimum score of 0 and a maximum score of 45 (23). The GSRS questionnaire has a scale from 0 to 3 for symptom severity and a scale from 0 to 4 for symptom frequency for every question with a minimum of 0 and maximum of 12 points per question (24). All these questions are anchored at the high end with the most negative or lowest intensity feelings (e.g., extremely unpleasant, not at all) and with opposing terms at the low end (e.g., extremely pleasant, very high and extreme). The survey also contained questions regarding the use of antireflux medication postoperatively and on re-operation.

\section{Statistical analysis}

All data are presented as mean and s.e.m. for normally distributed data and as median and interquartiles for abnormally distributed data. Comparisons were performed using the Wilcoxon signedranked test, the Mann-Whitney $U$ test, the $\chi^{2}$ test, and a dependent or an independent $t$-test as appropriate. $P<0.05$ was regarded as significant. All statistical analyses were performed using commercially available computer software (IBM Corp. Released 2012. IBM SPSS Statistics for Windows, Version 21.0. Armonk, NY: IBM Corp.)

\section{RESULTS}

\section{Baseline characteristics}

The vagus nerve injury group contained 23 patients $(11$ males, mean age 51 (range: 21-77) years, mean follow-up 19 (range: 14-25) years). The vagus nerve intact group consisted of 102 patients (49 males, mean age 48 (range: 19-73) years, mean followup 19 (range: 14-24) years). There were no statistically significant differences between these groups with respect to age, gender, and duration of follow-up. Before surgery, patients underwent gastric emptying testing, esophageal manometry, 24-h pH-monitoring, and vagus nerve integrity testing. Preoperative characteristics with respect to symptoms, acid exposure time, LES pressure, vagus nerve integrity testing, or gastric emptying were not significantly different between the vagus nerve injury and the vagus nerve intact group. The results of the preoperative work-up are listed in Table 2.

\section{Short-term outcome}

Vagus nerve integrity. Before surgery, none of the 125 patients had evidence of vagus nerve dysfunction. The plasma PP peak increase in response to insulin-induced hypoglycemia had a median value of $143(100-193) \mathrm{pmol} / \mathrm{l}$. After surgery the median value in the vagus nerve injury group was 17 (5-31) $\mathrm{pmol} / \mathrm{l}$ compared with $113(83-170) \mathrm{pmol} / \mathrm{l}$ in the vagus nerve intact group (Figure 2). Postoperative plasma PP response was compatible with truncal vagotomy (PP peak increase $\leq 47 \mathrm{pmol} / \mathrm{l}$ ) in 23 patients. With respect to the surgical procedure, 47 patients underwent Nissen fundoplication (13\% vagus nerve injury), 47 patients underwent Toupet hemi-fundoplication (17\% vagus nerve injury), and 31 underwent BM IV fundoplication (29\% vagus nerve injury) No significant difference in the distribution of vagus nerve injury was seen among the type of operations. We divided the patient population into two groups: one group with and one group without vagus nerve injury.

\section{Gastric emptying. Solids}

Gastric emptying of solids increased significantly after ARS for patients in the vagus nerve intact group. In the vagus nerve injury group, gastric emptying of solids did not change significantly after 
Table 2. Outcomes of 24-h pH-monitoring, esophageal manometry, and gastric emptying in patients with and without vagus nerve injury before and 6 months after antireflux surgery

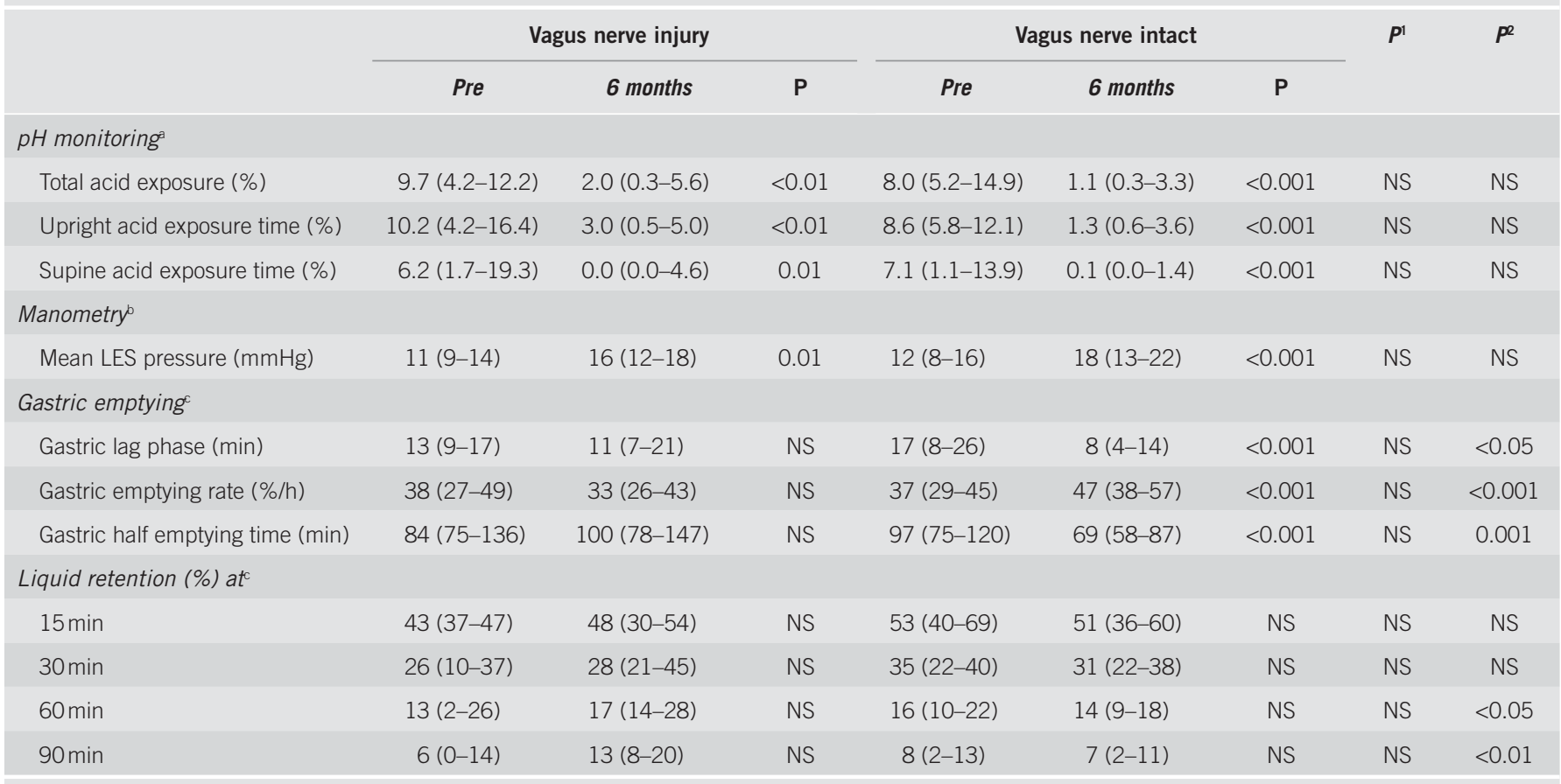

LES, lower esophageal sphincter; NS, not significant.

$P^{1}$ Comparison of baseline characteristics of both groups. $P^{2}$ comparison of postoperative outcomes of both groups. Presented as median (25-75\%).

${ }^{a} \mathrm{pH}$-monitoring $n=19 / 63$.

${ }^{b}$ Manometry $n=21 / 72$.

'Gastric emptying $n=13 / 51$.

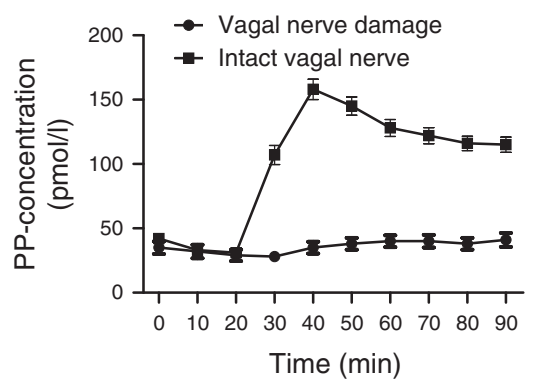

Figure 2. Difference between vagus nerve injury and the vagus nerve intact group 6 months after surgery for the hypoglycemia-induced pancreatic polypeptide test. At time $0 \mathrm{~min}$, insulin $(0.1 \mathrm{U} / \mathrm{kg})$ was administered.

ARS compared with preoperatively. The groups were not significantly different with respect to emptying parameters in the preoperative state; however, after ARS lag phase, emptying rate and half emptying time were significantly delayed in the patients with vagus nerve injury when compared with the vagus nerve intact group (Figure 3).

\section{Liquids}

No differences in gastric emptying of liquids were observed apart from a minor but statistically significant difference in reten- tion of liquids at 60 and $90 \mathrm{~min}$ postoperative between vagus nerve injury and vagus nerve intact patients (Table 2).

\section{$24 \mathrm{~h} p H$-monitoring and esophageal manometry}

ARS significantly reduced acid exposure time and increased the mean LES pressure 6 months after the procedure (Table 2). No statistically significant differences were found between both groups.

\section{Reflux symptoms}

Prior and 6 months after ARS, patients filled in a standard symptom questionnaire. The prevalence of symptoms after ARS was higher in the vagus nerve injury group. However, this did not reach statistical significance (Figure 4).

\section{Long-term outcome}

Responders. Of the 110 patients who received questionnaires via a letter, completed questionnaires were received from 71 patients (65\%). Thirty-four patients did not respond, four cases were excluded from the study because of an incorrect address, and 1 patient did not fill out the questionnaires correctly (Figure 1). Of these 71 patients, 13 (18\%) had vagus nerve injury, and 58 (82\%) had an intact vagus nerve.

Heartburn-GERD-HRQL. For the assessment of reflux symptoms $>10$ years after surgery, the GERD-HRQL questionnaire was 

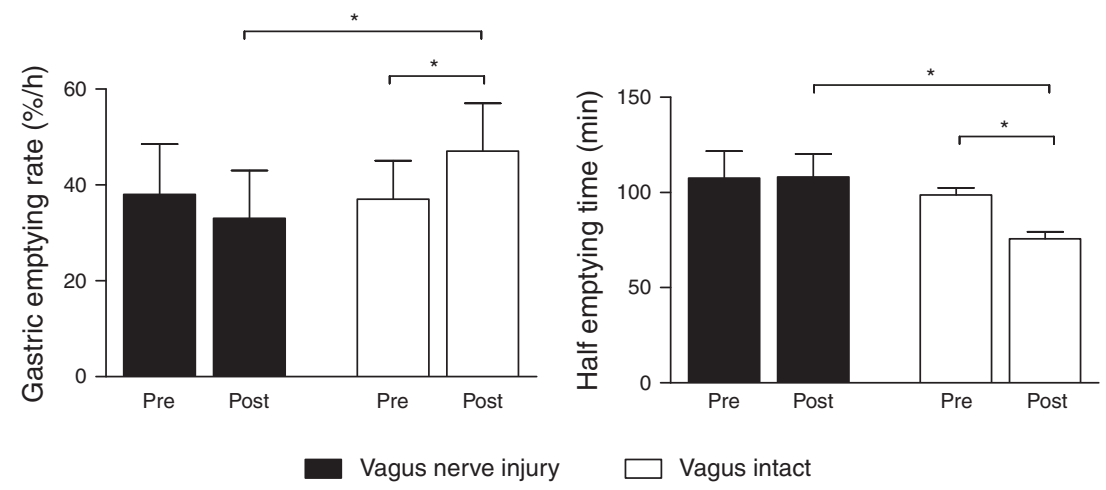

Figure 3. Comparison between vagus nerve injury and vagus nerve intact group for the difference in gastric emptying rate (\%/h; left panel) and gastric half emptying time (min; right panel) 6 months post-surgery. ${ }^{*} P<0.001$.

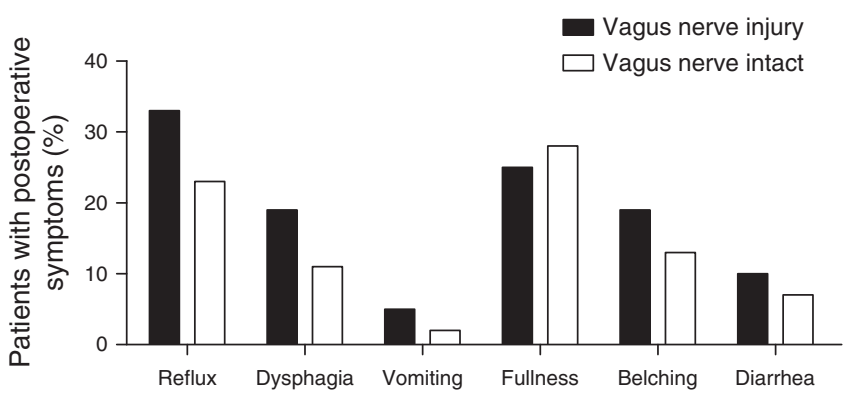

Figure 4. Difference in symptomatic outcome 6 months after surgery between the vagus nerve injury and the vagus nerve intact group.

used. Comparison between groups showed higher scores in the vagus nerve injury group (Table 3).

Dysphagia, regurgitation, gas-bloating, and cough. Severity and frequency of postoperative dysphagia, regurgitation, gas-bloating, and cough were assessed for both groups using the GSRS questionnaire. Similar results for all symptoms were shown in both groups (Table 3).

Satisfaction. For the assessment of general satisfaction, the GERDHRQL was used. Three categories for satisfaction, "satisfied", "neutral", and "unsatisfied", were described at the end of the questionnaire. Of the total group of 71 patients that responded 14-25 years after their antireflux surgery, 62\% was satisfied, 23\% was neutral, and $15 \%$ was unsatisfied. In the vagus nerve injury group $33.3 \%$ of patients were satisfied, $33.3 \%$ neutral and $33.3 \%$ unsatisfied. In the vagus nerve intact group $68 \%$ were satisfied, $21 \%$ neutral and $11 \%$ unsatisfied. A statistical significant difference was found between the two groups for the satisfaction rate (Table 3).

Proton-pump inhibitor usage. Of the 71 patients, $42 \%(n=30)$ were using a PPI after fundoplication. The other 58\% $(n=41)$ had not used PPIs since surgery. In the vagus nerve injury group, 7 patients $(54 \%)$ restarted the PPI use; in the vagus nerve
Table 3. Long-term outcome of antireflux surgery in patients with and without vagus nerve injury

\begin{tabular}{lccc} 
& $\begin{array}{c}\text { Vagus nerve } \\
\text { injury }(\boldsymbol{n}=\mathbf{1 3})\end{array}$ & $\begin{array}{c}\text { Vagus nerve } \\
\text { intact }(\boldsymbol{n}=58)\end{array}$ & $\boldsymbol{P}$ \\
$\begin{array}{l}\text { GERD-HRQL questionnaire } \\
\text { Total score }\end{array}$ & $13(5-25)$ & $3(0-9)$ & $<0.001$ \\
Satisfaction rate (\%) & 33 & 68 & $<0.05$ \\
PPI use (\%) & 54 & 41 & NS \\
Re-operation rate (\%) & 54 & 16 & 0.007 \\
GSRS questionnaire & & & \\
Heartburn & $2(0-6)$ & $1(0-3)$ & NS \\
Regurgitation & $1(0-8)$ & $0(0-2)$ & NS \\
Abdominal distention & $3(1-6)$ & $1(0-6)$ & NS \\
Dysphagia & $2(0-5)$ & $1(0-6)$ & NS \\
Coughing & $2(0-6)$ & $2(0-6)$ & NS \\
\hline
\end{tabular}

HRQL, Health Related Quality of Life; GERD, gastroesophageal reflux disease; GSRS, Gastrointestinal Symptom Rating Scale; NS, not significant; PPI, proton-pump inhibitor.

intact group, 24 patients (41\%) had recommenced taking PPI (Table 3).

Re-operation rate. Re-operation rate in the vagus nerve injury group was significantly higher: 7 of 13 patients (54\%) underwent re-operation. Three patients were operated because of reflux recurrence, 3 patients because of dysphagia, and 1 patient had severe gastroparesis for which a BII resection of the stomach was performed. In patients without vagus nerve injury, 9 out of 58 (16\%) patients underwent re-operation. Six patients were operated because of reflux recurrence, 1 patient because of dysphagia, 1 because of a slipped fundoplication, and 1 because of a rotated fundoplication. This difference in the re-operation rate between the two groups was statistically significant (Table 3 ). In addition, no significant difference in distribution of re-operation rate was seen among the different type of surgical procedures (Belsey Mark IV, laparoscopic Nissen, laparoscopic Toupet). 


\section{DISCUSSION}

In this short- and long-term follow-up of a prospective cohort, we evaluated the impact of vagus nerve injury on reflux control after antireflux surgery. The prevalence of vagus nerve injury tested by IH-PP was $20 \%$ in patients who underwent ARS. Our short-term data (6 months) indicate that symptom control and reflux control were not different among patients with and without vagus nerve injury. Postoperative gastric emptying was significantly delayed in the vagus nerve injury vs. vagus intact group. Concerning the data at 20-year follow-up, a significantly worse outcome with respect to heartburn symptoms, satisfaction rate, and re-operation rate was seen in patients with vagus nerve injury. Our results indicate that vagus nerve injury during antireflux surgery negatively affects long-term reflux control.

Using the IH-PP test, we observed a high prevalence of vagus nerve injury after ARS. In a previous study, we reported on vagus nerve injury after partial fundoplication. In that study, the prevalence of vagus nerve injury after ARS was 10\% (14). It should be taken into account that the number of patients in that study was much smaller and that we evaluated only one single surgical technique and therefore that the study may not give an accurate estimate of the prevalence of vagus nerve injury after ARS in general-that is, after all types of ARS. In the present study, three different surgical techniques (BM IV, Toupet, and Nissen) have been employed and a large group of patients was included. In 23 patients, the results were compatible with vagus nerve injury: $13 \%(6 / 47)$ in the Nissen group, $18 \%(8 / 47)$ in the Toupet group, and 29\% (9/31) in the BM IV fundoplication group. We did not observe significant differences between surgical procedures with respect to the prevalence of vagus nerve injury.

Risk factors for vagus nerve injury after ARS have not been studied systematically. One may assume that the risk of vagus nerve injury is both procedure and surgeon related as operating and manipulating near the branches of the vagus nerve may easily lead to direct or indirect injury. An intrathoracic approach (BM IV) is technically more challenging and therefore more prone to vagus nerve injury. As mentioned earlier, we did not observe significant differences in vagus nerve dysfunction between the surgical procedures that have been employed in this study, although the percentage of patients with vagus nerve injury after BM IV was higher than after the two other procedures.

Other factors that potentially may increase a risk of vagus nerve injury during ARS include the presence of a large para-esophageal or axial hiatal hernia, previous abdominal surgery, severe esophagitis causing adhesions, poor visibility of the anatomy in the gastroesophageal region, anatomical variations of the vagus nerve, and limited experience with the procedure of the surgeon. Patients included in this study underwent antireflux surgery as a primary procedure and no para-esophageal hiatal hernia's were present. Furthermore, all patients were operated in a high volume center by surgeons experienced with the types of operations employed in this study.

The mechanisms by which vagus nerve injury negatively affects the outcome after ARS are not fully understood. The vagus nerve is known to have an important role in the motor and sensory regulatory functions of the esophagus and stomach. Vagus nerve injury may impair these functions and thereby lead to upper gastrointestinal symptoms.

Previous studies have established an association between vagus nerve injury after ARS and impaired gastric motility, resulting in delayed gastric emptying or even gastroparesis (25-27). In more detail, impairment of the gastric accommodation of the proximal stomach, hypomotility of the antrum (with loss of trituration and retention of solids), and loss of pyloric relaxation (with stasis) have been associated with vagotomy and conditions after gastric surgery (28-34).

Longer retention of food in the stomach leads to higher intragastric pressures and thereby may promote reflux even in the presence of a restored esophago-gastric barrier. Recently, Gourcerol et al. showed that, in GERD patients with delayed gastric emptying, the number of postprandial reflux events measured with $\mathrm{pH}$-impedance monitoring was increased, with a longer bolus clearance time and more proximal esophageal extension of the refluxate. This was also true for reflux symptoms in patients with delayed gastric emptying compared with patients with normal gastric emptying (35). Rebecchi et al. evaluated the association between gastric emptying with long-term reflux control in GERD patients after ARS. These authors showed that delayed gastric emptying was strongly associated with poor reflux control (36). Taken together, these findings indicate that delayed gastric emptying contributes to GERD and that it may negatively influence the effect of ARS.

We observed that gastric emptying improved for solids but not for liquids after ARS in the vagus nerve intact group, whereas it remained unchanged both for liquids and solids in the vagus nerve injury group at 6 months after surgery.

Delayed gastric emptying is seen in up to $40 \%$ of patients with GERD (37). Fundoplication is known to accelerate gastric emptying (14,38). In ARS, part of the fundus is utilized for creating the fundoplication and thereby reducing postprandial gastric volume and gastric capacity. This results in a higher proximal gastric tone and reduces duration of postprandial relaxation of the proximal stomach, leading to propulsion of food into the antrum with acceleration in gastric emptying (38-40). Acceleration of gastric emptying after fundoplication may positively influence the antireflux effect of the procedure. The delay in liquid emptying at 60 and 90 min seen after ARS in the vagus nerve injury group vs. vagus nerve intact group is not in line with literature data. It has been indicated that vagotomy leads to a reduction in fundus accommodation and body of the stomach and thereby an acceleration of liquid emptying (26,41-43). In our study, testing of solid and liquid gastric emptying was conducted simultaneously, with potential interference of the solid and liquid components.

We confirmed findings from previous studies and extended there upon: vagus nerve injury did not negatively affect outcomes 6 months after ARS despite the persistence of delayed gastric emptying $(14,15,17)$. However, during long-term follow-up, a worse outcome of reflux control was found in the vagus nerve 
injury group. A possible explanation for the difference in control of reflux symptoms during short- and long-term follow-up is not readily available. One option is that the newly created gastroesophageal valve may gradually lose efficacy with respect to its reflux barrier over time (44). Increasing age with loss of compliance and elasticity of tissue may lead to reduced efficacy of the gastroesophageal valve.

The re-operation rate differed significantly between both groups. Over $50 \%$ of patients in the vagus nerve injury group have been re-operated. Most patients underwent re-operation because of recurrence of reflux. In one patient from the vagus nerve injury group, severe gastroparesis had developed. This was so debilitating that a partial resection of the stomach had to be performed. Despite high re-operation rates, symptoms associated with GERD persisted in this patient group. The findings of our study suggest that patients who have recurrent reflux symptoms following ARS might benefit from vagal integrity testing before deciding on additional treatment, as a normal outcome of gastric emptying testing does not appear to exclude vagal nerve injury. Therefore, we believe careful and objective evaluation of symptoms and measurement of gastric emptying and PP-IH testing after ARS is warranted before considering re-operation in these patients.

Some limitations to this study should be addressed. The longterm symptomatic outcome cannot be directly compared with the 6-month data because other types of questionnaires were used. Furthermore, not all patients underwent all tests such as gastric emptying test, esophageal manometry, and 24-h pH-monitoring pre- and 6 months after surgery.

The strength of our study is the evaluation of vagus nerve integrity by IH-PP measurements both before and after fundoplication in all 125 patients so that we are certain that the outcome of the test when pointing to vagus nerve dysfunction was related to the surgical procedure. To our best knowledge, our study is the largest to date reporting on vagus nerve dysfunction after ARS with long-term results.

In conclusion: vagus nerve injury occurs in up to $20 \%$ of patients who undergo antireflux surgery. Vagus nerve injury does not negatively affect short-term reflux control 6 months after antireflux surgery. However, long-term follow-up showed a negative effect on symptom control and a significantly higher re-operation rate in patients with vagus nerve injury. Preserving the main trunks of the vagus nerve is therefore of utmost importance in order to maintain vagus nerve integrity and contribute to reflux control after ARS.

\section{CONFLICT OF INTEREST}

Guarantor of the article: Adrian A.M. Masclee, MD, PhD. Specific author contributions: S. van Rijn: acquisition, analysis and interpretation of data, and drafting of manuscript. N.F. Rinsma: analysis of data and drafting of manuscript. M.Y.A. van HerwaardenLindeboom, J. Ringers, P.J.J. van Rijn, H.G. Gooszen, and R.A. Veenendaal: data acquisition. J.M. Conchillo: Approval of final manuscript. N.D. Bouvy: interpretation of data. A.A.M. Masclee: study concept, interpretation of data, drafting and approval of final manuscript, and overall study supervision.

Financial support: This study was conducted without funding. Potential competing interests: None.

\section{Study Highlights}

\section{WHAT IS CURRENT KNOWLEDGE}

Antireflux surgery (ARS) is an accepted alternative therapy for patients with symptoms refractory to proton-pump inhibitors (PPIs) or not willing to take lifelong PPIs.

Vagus nerve injury is a feared complication of ARS that may negatively affect reflux control.

\section{WHAT IS NEW HERE}

The prevalence of vagus nerve injury after ARS is $20 \%$.

At short-term follow-up, gastric emptying was significantly delayed in the vagus nerve injury group vs. the vagus nerve intact group.

At short-term follow-up, reflux control was similar between both groups.

Long-term follow-up showed significantly less reflux control and a higher re-operation rate in the vagus nerve injury group.

\section{REFERENCES}

1. Dent J, El-Serag HB, Wallander MA et al. Epidemiology of gastro-oesophageal reflux disease: a systematic review. Gut 2005;54:710-7.

2. Dallemagne B, Weerts JM, Jehaes C et al. Laparoscopic Nissen fundoplication: preliminary report. Surg Laparosc Endosc 1991;1:138-43.

3. Dallemagne B, Perretta S. Twenty years of laparoscopic fundoplication for GERD. World J Surg 2011;35:1428-35.

4. Wang YR, Dempsey DT, Richter JE. Trends and perioperative outcomes of inpatient antireflux surgery in the United States, 1993-2006. Dis Esophagus 2011;24:215-23.

5. Broeders JA, Mauritz FA, Ahmed Ali U et al. Systematic review and metaanalysis of laparoscopic Nissen (posterior total) versus Toupet (posterior partial) fundoplication for gastro-oesophageal reflux disease. Br J Surg 2010;97:1318-30.

6. Papasavas P. Functional problems following esophageal surgery. Surg Clin North Am 2005;85:525-38.

7. Broeders JA, Sportel IG, Jamieson GG et al. Impact of ineffective oesophageal motility and wrap type on dysphagia after laparoscopic fundoplication. Br J Surg 2011;98:1414-21.

8. Kessing BF, Broeders JA, Vinke $\mathrm{N}$ et al. Gas-related symptoms after antireflux surgery. Surg Endosc 2013;27:3739-47.

9. Lindeboom MY, Ringers J, Straathof JW et al. The effect of laparoscopic partial fundoplication on dysphagia, esophageal and lower esophageal sphincter motility. Dis Esophagus 2007;20:63-8.

10. Kozarek RA, Low DE, Raltz SL. Complications associated with laparoscopic anti-reflux surgery: one multispecialty clinic's experience. Gastrointest Endosc 1997;46:527-31.

11. Trus TL, Bax T, Richardson WS et al. Complications of laparoscopic paraesophageal hernia repair. J Gastrointest Surg 1997;1:221-7.discussion 228

12. Schwartz TW. Pancreatic polypeptide: a hormone under vagal control. Gastroenterology 1983;85:1411-25.

13. Schwartz TW, Holst JJ, Fahrenkrug J et al. Vagal, cholinergic regulation of pancreatic polypeptide secretion. J Clin Invest 1978;61:781-9.

14. Lindeboom MY, Ringers J, van Rijn PJ et al. Gastric emptying and vagus nerve function after laparoscopic partial fundoplication. Ann Surg 2004; 240:785-90.

15. DeVault KR, Swain JM, Wentling GK et al. Evaluation of vagus nerve function before and after antireflux surgery. J Gastrointest Surg 2004;8: 883-8.discussion 888-889.

16. Straathof JW, Ringers J, Masclee AA. Prospective study of the effect of laparoscopic Nissen fundoplication on reflux mechanisms. Br J Surg 2001;88: 1519-24.

17. Oelschlager BK, Yamamoto K, Woltman T et al. Vagotomy during hiatal hernia repair: a benign esophageal lengthening procedure. J Gastrointest Surg 2008;12:1155-62. 
18. Skinner DB, Belsey RH. Surgical management of esophageal reflux and hiatus hernia. Long-term results with 1,030 patients. J Thorac Cardiovasc Surg 1967;53:33-54.

19. Nissen R. [A simple operation for control of reflux esophagitis]. Schweiz Med Wochenschr 1956;86:590-2.

20. Toupet A. [Technic of esophago-gastroplasty with phrenogastropexy used in radical treatment of hiatal hernias as a supplement to Heller's operation in cardiospasms]. Mem Acad Chir (Paris) 1963;89:384-9.

21. Masclee AA, de Best AC, de Graaf R et al. Ambulatory 24-hour pH-metry in the diagnosis of gastroesophageal reflux disease. Determination of criteria and relation to endoscopy. Scand J Gastroenterol 1990;25:225-30.

22. Lamers CB, Diemel CM, van Leer E et al. Mechanism of elevated serum pancreatic polypeptide concentrations in chronic renal failure. J Clin Endocrinol Metab 1982;55:922-6.

23. Velanovich V. The development of the GERD-HRQL symptom severity instrument. Dis Esophagus 2007;20:130-4.

24. Allen CJ, Parameswaran K, Belda J et al. Reproducibility, validity, and responsiveness of a disease-specific symptom questionnaire for gastroesophageal reflux disease. Dis Esophagus 2000;13:265-70.

25. Camilleri M, Parkman HP, Shafi MA et al. Clinical guideline: management of gastroparesis. Am J Gastroenterol 2013;108:18-37.quiz 38.

26. Jamieson GG, Maddern GJ, Myers JC. Gastric emptying after fundoplication with and without proximal gastric vagotomy. Arch Surg 1991;126:1414-7.

27. Olinde AJ, Maher JW, McGuigan JE et al. The effect of fundoplication with or without proximal gastric vagotomy on gastric emptying and serum gastrin. Am Surg 1985;51:690-2.

28. Shafi MA, Pasricha PJ. Post-surgical and obstructive gastroparesis. Curr Gastroenterol Rep 2007;9:280-5.

29. Behrns KE, Sarr MG. Diagnosis and management of gastric emptying disorders. Adv Surg 1994;27:233-55.

30. Miyano Y, Sakata I, Kuroda K et al. The role of the vagus nerve in the migrating motor complex and ghrelin- and motilin-induced gastric contraction in suncus. PLoS One 2013;8:e64777.

31. Fich A, Neri M, Camilleri M et al. Stasis syndromes following gastric surgery: clinical and motility features of 60 symptomatic patients. J Clin Gastroenterol 1990;12:505-12.
32. Azpiroz F, Malagelada JR. Gastric tone measured by an electronic barostat in health and postsurgical gastroparesis. Gastroenterology 1987;92: 934-43.

33. Bredenoord AJ, Chial HJ, Camilleri $\mathrm{M}$ et al. Gastric accommodation and emptying in evaluation of patients with upper gastrointestinal symptoms. Clin Gastroenterol Hepatol 2003;1:264-72.

34. Lagoo J, Pappas TN, Perez A. A relic or still relevant: the narrowing role for vagotomy in the treatment of peptic ulcer disease. Am J Surg 2014;207: 120-6.

35. Gourcerol G, Benanni Y, Boueyre E et al. Influence of gastric emptying on gastro-esophageal reflux: a combined $\mathrm{pH}$-impedance study. Neurogastroenterol Motil 2013;25:800-e634.

36. Rebecchi F, Allaix ME, Giaccone $\mathrm{C}$ et al. Gastric emptying as a prognostic factor for long-term results of total laparoscopic fundoplication for weakly acidic or mixed reflux. Ann Surg 2013;258:831-6.discussion 836-837.

37. Gonlachanvit S, Maurer AH, Fisher RS et al. Regional gastric emptying abnormalities in functional dyspepsia and gastro-oesophageal reflux disease. Neurogastroenterol Motil 2006;18:894-904.

38. Vu MK, Straathof JW, v d Schaar PJ et al. Motor and sensory function of the proximal stomach in reflux disease and after laparoscopic Nissen fundoplication. Am J Gastroenterol 1999;94:1481-9.

39. Vu MK, Ringers J, Arndt JW et al. Prospective study of the effect of laparoscopic hemifundoplication on motor and sensory function of the proximal stomach. Br J Surg 2000;87:338-43.

40. Lindeboom MY, Vu MK, Ringers J et al. Function of the proximal stomach after partial versus complete laparoscopic fundoplication. Am J Gastroenterol 2003;98:284-90.

41. Van Hee R, Mistiaen W, Block P. Gastric emptying of liquids after highly selective vagotomy for duodenal ulcer. Hepatogastroenterology 1989;36: 92-6.

42. Stadaas JO. Effect of vagotomy on gastric motility. Scand J Gastroenterol Suppl 1976;42:85-8.

43. Gleysteen JJ, Burdeshaw JA, Hallenbeck GA. Gastric emptying of liquids after different vagotomies and pyloroplasty. Surg Gynecol Obstet 1976;142:41-8.

44. Robinson B, Dunst CM, Cassera MA et al. 20 years later: laparoscopic fundoplication durability. Surg Endosc 2014;29:2520-4. 